\title{
Personennahe Dienstleistung der Zukunft
}

\section{Mehr digital ist nicht weniger Mensch}

\author{
Susanne Robra-Bissantz $\cdot$ Christoph Lattemann
}

Ganz harmlos haben wir, wie immer mit großem Vorlauf, die vorliegende Ausgabe der HMD - Praxis der Wirtschaftsinformatik angestoßen. Denn auch die personennahe Dienstleistung wird zunehmend digital. Die personennahe Dienstleistung, die wir als Dienstleistung charakterisieren, die einen Menschen in seinem Leben unterstützt. Egal, ob direkt, in der Pflege oder Kinderbetreuung, aber auch, wenn es beispielsweise um seine Wohnung geht, wie in der Unterstützung bei handwerklichen Problemen, oder wenn man über den Kauf eines Produktes sein Leben verbessern möchte.

In den letzten Monaten und insbesondere mit Ihren Beiträgen ist, so sehen wir das, die personennahe Dienstleistung sozusagen über sich hinausgewachsen. Denn es wird in Forschung und Praxis immer deutlicher, dass es genau das Merkmal „personennah“ ist, das die Zukunft der digitalen Transformation treibt. In unserem, gemeinsam mit Herrn Ziegler (PTKA), dem Begleiter einer entsprechenden Förderlinie des BMBF, verfassten Grundlagenartikel Die Komposition personennaher Dienstleistungen von morgen zeigen wir auf, dass es in Zukunft hinsichtlich der Digitalisierung nicht reichen wird, in möglichst effizient bereitgestellten Produkten für Märkte zu denken. Und diese Produkte durch Dienstleistungen zu ergänzen, verharrt in Dienstleistungen, die produktnah - und eben nicht personennah sind. Aber das Spiel hat sich geändert. Über die zu jedem einzelnen Menschen reichende digitale Vernetzung wird die einzelne Person zum aktiven Partner, der mit einem Wischen am Mobiltelefon entscheidet, wie er sich sein Leben besser machen möchte. Statt

\footnotetext{
S. Robra-Bissantz $(\square)$

Technische Universität Braunschweig, Universitätsplatz 2, 38106 Braunschweig, Deutschland

E-Mail: s.robra-bissantz@tu-braunschweig.de

C. Lattemann

Jacobs University Bremen, Campus Ring 1, 28759 Bremen, Deutschland

University of Agder, Universitetsveien 25, 4630 Kristiansand, Norwegen
} 
Effizienz im Unternehmen steht dann der Wert für den privaten Akteur, für den Kunden, im Vordergrund: er wählt das Produkt oder besser: diejenige Dienstleistung, die unter Umständen ein Produkt umfasst, die ihm den höchsten Wert verspricht. Das erfordert ein Umdenken, das sich auch im Design dieser digital unterstützten Dienstleistungen niederschlagen muss: ausgehend vom Kunden (personennah), mit Fokus auf Interaktionen und Kooperationen. Dabei führt dieser Beitrag, ebenso wie weitere Beiträge dieser Ausgabe, seine Erkenntnisse auf die neue betriebswirtschaftliche Service Logik zurück, die theoretisch aufzeigen kann, wie sich die heutige Geschäftstätigkeit ändert.

Insgesamt passen die konsequente Serviceorientierung und Digitalisierung gut zusammen. Mehr von der Person aus an ihre Bedürfnisse zu denken, lässt sich häufig durch Digitalisierung befördern oder ermöglichen. So zeigt der Beitrag Digitalisierung als Grundlage wertvoller Zusammenarbeit auf, dass es über digitale Zusammenarbeit gelingt, zum einen den Kunden als kooperierenden Partner näher an sich zu binden und ihm zum anderen zusammen mit weiteren kooperierenden Unternehmen eine gute Dienstleistung zu bieten. Dieser Beitrag führt zudem eine Reihe von Beispielen aus Projekten des BMBF zusammen, die sich bereits seit 1,5 Jahren konsequent mit der personennahen Dienstleistung beschäftigen und zum Teil ebenfalls in dieser Ausgabe der HMD zu finden sind.

Dazu gehört bereits der nächste Artikel (Möglichkeiten der Digitalisierung zur Beratung von hochkonfigurierbaren und hochindividualisierbaren Gütern), der für die Möbelbranche aufzeigt, wie eine Digitalisierung der personennahen Beratungsleistung mithilfe von Augmented Reality über ethnographische Studien genau auf die Verkaufssituation eingeht und wichtige Bedürfnisse des Menschen in der Visualisierung seiner Konfiguration aber zugleich in der Beziehung zum Verkäufer, wie beispielsweise Reziprozität und Vertrauen, gewährleisten kann. Mehr digital ist nicht weniger Mensch. Vielleicht sogar mehr.

Beispielsweise, wenn neue analysebasierte Dienstleistungen ihren Kunden ermöglichen bessere Entscheidungen zu treffen oder komplexere Probleme zu lösen. Der Beitrag Die Gestaltung analysebasierter Dienstleistungen zeigt hier im Sinne einer modernen Wirtschaftsinformatik auf, wie im Unternehmen schrittweise das Design der bereits breit diskutierten aber schwer zu konzipierenden Dienstleistungen gelingt, die vorhandene Daten aus vorhergehenden Interaktionen und Prozessen nutzen und gewinnbringend anbieten.

In den folgenden vier Artikeln geht es um die Gestaltung von personennahen Dienstleistungen in ganz besonderen Bereichen. Gemein ist ihnen, dass sie konsequent vom Nutznießer der Dienstleistung ausgehen - und dabei zunächst aufzeigen, dass es beispielsweise in der Forschung wirklich wichtig ist, eine Unterstützung von Forschenden in Projekten auch personennah zu sehen (Des Schusters Schuhe. Über die Notwendigkeit der Betrachtung digitaler personennaher Dienstleistungen für Forschende)! Es erscheint im Nachhinein völlig offensichtlich, dass die Unterstützung, beispielsweise von den entsprechenden Service-Abteilungen in Universitäten oder Unternehmen, sich daran ausrichten muss, wann sie für den Forschenden wirklich wertvoll - und damit eine Dienstleistung ist. Auch die betriebliche Weiterbildung sollte sich, so der Beitrag Dem Mitarbeiter zu Diensten. Weiterbildung und Qualifizierung als Personennahe Dienstleistung, daran ausrichten, wie sie ganz indi- 
viduell die Probleme der Mitarbeiter aufgrund mangelnder Kompetenzen lösen kann und diese dabei am Besten in die Entwicklung der Weiterbildungsdienstleistung für sie einbezieht.

Das gemeinsame Problemlösen zwischen Kunde und Anbieter der Dienstleistung wird als Co-Creation bezeichnet: der Kunde löst sein Problem und der Anbieter hilft ihm, oder der Kunde wirkt als Prosumer an der Dienstleistung mit. Sie eröffnet, als neue Sichtweise, neue Optionen. Beispielsweise, wenn Mitarbeiter gegenseitig für Kollegen die Lerninhalte im Bereich der Weiterbildung erstellen (Co-Creation durch Peers im digitalen Lernen). Neben den Bedürfnissen des Lernenden sind dann auch diejenigen des Erstellers zu berücksichtigen und ein entsprechendes Design schlägt vor, dass hier ein Chatbot über seine Interaktionen zu Motivation und Veranwortungsbewusstsein beitragen kann.

Im Artikel Do-It-Yourself-Plattformen für individuelle Wearables ist die Idee, dem Kunden sehr viel eigene Gestaltung zu überlassen. Dabei wird er durch eine digitale personennahe Dienstleistung in seiner schwierigen Aufgabe unterstützt: über digitale Konfigurations- und Matchingsysteme, aber auch über eine Community von anderen Kunden und Dienstleistern. Beide letzteren Beiträge sind gute Beispiele dafür, wie verschiedenste Partner aber auch digitale Komponenten Aufgaben in der Dienstleistung übernehmen.

Die Konstellation, auf der sich Menschen in der Rolle von Anbieter oder Nachfrager über eine digitale Plattform koordinieren, ist sehr typisch für die personennahe Dienstleistung. In den nächsten zwei Beiträgen wird dies im Bereich von haushaltsnahen Dienstleistungen diskutiert. Der Beitrag Incentivierung von Prosumern auf einem elektronischen Marktplatz für personennahe Dienstleistungen berücksichtigt dabei den Anbieter von nachbarschaftlichen Leistungen und analysiert über verschiedene theoretische Ansätze, wie man seine Barrieren, wie z.B. das Gefühl mangelnder Kompetenz, überwinden oder seine Motivation, z. B. über Badgets, steigern kann. Im Beitrag Die Digitalisierung von Personenbezogenen Dienstleistungen durch Online-Plattformen: Woher kommt die geringe Nutzung? ist umfassend, auch in einer Welt ohne digitale Plattformen, theoretisch und in qualitativen Interviews analysiert, warum Dienstleistungen, in der Kinderbetreuung, als Haushaltshilfe oder im Garten noch immer selten (digital) in Anspruch genommen werden. Es leiten sich in beiden Fällen erste Empfehlungen für die digitale Dienstleistung der Plattform ab.

Letztendlich ist es das Vertrauen, in den jeweiligen Dienstleister oder auch in die Plattform, das häufig den Ausschlag über den Erfolg, beispielsweise einer Nachbarschaftshilfe, gibt. Denn die personennahe Dienstleistung, wie eine Kinderbetreuung, gibt hier Grund zur Sorge. Damit beschäftigen sich die folgenden zwei Beiträge. Vertrauensbasierte Organisationen als Grundlage von erfolgreichen digitalen Plattformen für personennahe Dienstleistungen beschreibt für die Nachbarschaftshilfe, dass die Organisation, die hinter der Plattform steht, gemeinwohl- oder gewinnorientiert, Einfluss auf die Intention der Akteure hat, diese zu nutzen. Dazu tritt, wie bereits in vorherigen Beiträgen aufgezeigt, inwieweit die Nutzer in die Gestaltung der Dienstleistungen einbezogen werden.

Online-Plattformen und Personennahe Dienstleistungen: Eine explorative Studie über vertrauensbildenden Maßnahmen zeigt über die Analyse von mehr als 100 
Plattformen mit personennahen Dienstleistungen auf, welche vertrauensbildenden Mechanismen, für die peer-2-peer Kinderbetreuung erfolgversprechend sind. Diese reichen von der Darstellung von umfangreichen Informationen über die Partner und Kommunikation zwischen ihnen bis hin zu aufwändigen technischen Lösungen, wie einem Notfallknopf.

Mit dem Beitrag SOBRADO - Der Aufbau einer Transaktionsplattform für Versicherungsbroker und Versicherer wird für eine Plattform im Versicherungsmarkt theoretisch wohl fundiert und am Praxisbeispiel aufgezeigt, wie die Gestaltung beispielsweise im Umgang mit Netzeffekten, kritischen Massen oder Preismodellen gelingt.

Abschließend zeigt der Beitrag Digitalisierung personennaher Dienstleistungen in der Kinder- und Jugendhilfe auf, wie auch bei personennahen Dienstleistungen, die weiterhin ganz menschlich erbracht werden sollen, die Digitalisierung den Menschen von seinen, genau zu definierenden, aufwändigen, automatisierbaren Aktivitäten entlasten kann.

Wir danken ganz herzlich den Verbundprojekten der Förderlinie „Personennahe Dienstleistungen“" des BMBF, die mit insgesamt acht Artikeln zu unserem Heft beigetragen haben. Falls Sie mehr über diese Förderlinie erfahren wollen, finden Sie alle Informationen unter www.bedien.org.

Heute sind wir ganz fasziniert davon, wieviele, für eigentlich alle Unternehmen wichtige Themen in der vorliegenden Ausgabe unserer Zeitschrift angesprochen und diskutiert sind. Und wir hoffen, es geht Ihnen ähnlich.

Funding Open Access funding provided by Projekt DEAL.

Open Access Dieser Artikel wird unter der Creative Commons Namensnennung 4.0 International Lizenz veröffentlicht, welche die Nutzung, Vervielfältigung, Bearbeitung, Verbreitung und Wiedergabe in jeglichem Medium und Format erlaubt, sofern Sie den/die ursprünglichen Autor(en) und die Quelle ordnungsgemäß nennen, einen Link zur Creative Commons Lizenz beifügen und angeben, ob Änderungen vorgenommen wurden.

Die in diesem Artikel enthaltenen Bilder und sonstiges Drittmaterial unterliegen ebenfalls der genannten Creative Commons Lizenz, sofern sich aus der Abbildungslegende nichts anderes ergibt. Sofern das betreffende Material nicht unter der genannten Creative Commons Lizenz steht und die betreffende Handlung nicht nach gesetzlichen Vorschriften erlaubt ist, ist für die oben aufgeführten Weiterverwendungen des Materials die Einwilligung des jeweiligen Rechteinhabers einzuholen.

Weitere Details zur Lizenz entnehmen Sie bitte der Lizenzinformation auf http://creativecommons.org/ licenses/by/4.0/deed.de. 